\title{
Crosstalk between keratinocytes and immune cells in inflammatory skin diseases
}

\author{
Xinhui Ni, Yuping Lai* ${ }^{*}$ \\ Shanghai Frontiers Science Center of Genome Editing and Cell Therapy, Shanghai Key Laboratory of Regulatory Biology, School \\ of Life Sciences, East China Normal University, Shanghai 200241, China
}

*Correspondence: Yuping Lai, Shanghai Frontiers Science Center of Genome Editing and Cell Therapy, Shanghai Key Laboratory of Regulatory Biology, School of Life Sciences, East China Normal University, 500 Minhang DongChuan Road, Shanghai 200241, China. yplai@bio.ecnu.edu.cn

Academic Editor: Masutaka Furue, Kyushu University, Japan

Received: August 12, 2021 Accepted: December 1, 2021 Published: December 31, 2021

Cite this article: Ni X, Lai Y. Crosstalk between keratinocytes and immune cells in inflammatory skin diseases. Explor Immunol. 2021;1:418-31. https://doi.org/10.37349/ei.2021.00028

\begin{abstract}
Cutaneous homeostasis is maintained by dynamic cellular communications between different cell types in the skin through interactions with various mediators, including cytokines, chemokines and antimicrobial peptides/proteins (AMPs). Keratinocytes, as the major cell type of the epidermis, not only form a passive physical barrier, but also actively participate in the pathogenesis of many, if not all, inflammatory skin diseases. Keratinocytes highly interact with immune cells to shape, amplify or regulate inflammatory responses, thus triggering and/or sustaining these inflammatory skin diseases. In this review, crosstalk between keratinocytes and immune cells is summarized, and its contributions to two major inflammatory skin disorders including psoriasis and atopic dermatitis are highlighted.
\end{abstract}

\section{Keywords}

Crosstalk, keratinocytes, immune cells, inflammatory skin diseases

\section{Introduction}

Skin is the first line of host defense against danger signals from the environment. Cutaneous homeostasis and skin defense are maintained by dynamic cellular communications between different cell types in the skin through interactions with various mediators, including cytokines, chemokines and antimicrobial peptides/ proteins (AMPs) [1]. Keratinocytes, as a major cell type of the epidermis in the skin, are derived from skin epithelial stem cells in the basal layer, and then differentiate into different keratinocyte layers above the basal zone, such as spinous, granular, and cornified layers [2]. To balance proliferation and epidermal thickness, keratinocytes undergo apoptosis and apoptotic keratinocytes contribute to stratum corneum (SC) formation [3]. Keratinocytes not only passively form a physical barrier against pathogens, but also can sense danger signals and initiate a primary inflammation to protect hosts. Moreover, keratinocytes are capable of responding to inflammatory mediators produced by immune cells to amplify or maintain inflammatory 
responses in the skin. Accumulating evidence has suggested that keratinocytes are actively involved in the pathogenesis of many, if not all, inflammatory skin diseases. We herein summarize the crosstalk between keratinocytes and immune cells, and highlight its contributions to two major inflammatory skin disorders including psoriasis and atopic dermatitis (AD).

\section{Crosstalk between keratinocytes and immune cells in psoriasis}

Psoriasis is a common chronic inflammatory skin disease characterized by a considerable thick epidermis and a dense dermal infiltration of immune cells [4]. The debate about which cells, keratinocytes or immune cells, trigger psoriasis lasts over two decades. Undoubtedly, no matter who drives psoriasis, the crosstalk between keratinocytes and immune cells plays essential roles during the development of psoriasis [5]. Here we will discuss two different hypotheses about how the crosstalk between keratinocytes and immune cells drives psoriasis.

\section{Keratinocytes initiate immune responses in psoriasis}

Early in 1991, Nickoloff [6] proposed the idea of the "cytokine network" in psoriasis. In this "cytokine network" hypothesis, keratinocytes and inflammatory cells, such as T cells and dendritic antigen-presenting cells, interact with each other to drive cutaneous inflammation, and keratinocytes were indicated to act as "signal transducers" to convert exogenous stimuli into the production of cytokines, adhesion molecules, and chemotactic factors responsible for initiation of "antigen-independent" cutaneous inflammation $[7,8]$. The observation from Haase group that mice with the specific ablation of inhibitor kappa B kinase 2 (IKK2) in keratinocytes (K14-Cre/Ikk2 $2^{F L / F L}$ ) spontaneously developed psoriasis-like pathologies in $\alpha \beta$ T-cell-independent way by upregulating of tumor necrosis factor (TNF) receptor 1 (TNFR1)-dependent interleukin 24 (IL-24) and activating signal transducer and activator of transcription 3 (STAT3) in keratinocytes $[9,10]$ also suggests that the keratinocytes can initiate inflammatory responses in psoriasis. The latter discovery that inducible epidermal deletion of JunB/c-Jun in adult mice and Rag2-deficient JunB/c-Jun double-mutant mice developed a phenotype resembling the pathology of psoriasis [11] confirmed this hypothesis. Moreover, several groups demonstrated that genetic defects of caspase recruitment domain family member 14 (CARD14) [12-15] in keratinocytes can directly cause psoriasis via regulating IL-17A-induced mitogen-activated protein kinase (MAPK) and nuclear factor kappa B (NF-kB) activation to produce pro-inflammatory factors [16]. These observations suggest that keratinocytes can be considered as a trigger of cutaneous inflammation in psoriasis, and cytokines produced by keratinocytes play key roles in the initiation of inflammation in psoriasis.

In 2007, Gilliet and colleagues $[17,18]$ confirmed that keratinocytes initiated the onset of psoriasis via secreting the AMPs LL-37 (also called hCAP18) conjugated with self-DNA or RNA to activate Toll-like receptor 9 (TLR9) or TLR7/8 in plasmacytoid dendritic cells (pDCs) after skin injury. Activated pDCs released interferon alpha (IFN $\alpha$ )/IFN $\beta$ to trigger initial activation of myeloid dendritic cells (mDCs) and IL-17-producing T cells $[18,19]$. Activated IL-17-producing $\mathrm{T}$ cells were then recruited into lesional skin and produced multiple cytokines such as IL-17 and IL-22 to establish a cytokine milieu that dictates specific gene signatures in keratinocytes, which, thus, overexpress several inflammatory mediators to amplify local immune reactions [20-22].

After that, our group also found that skin injury activated TLR3 in keratinocytes to produce multiple pro-inflammatory cytokines, such as TNF, IL-6 and IL-36 [23, 24]. IL-36 in turn induced keratinocytes to produce the antimicrobial protein regenerating islet-derived protein 3 alpha (REG3A). REG3A acted back on keratinocytes to inhibit keratinocyte differentiation, thus inducing epidermal hyperplasia [25]. Furthermore, IL-36 induced the pathological IL-23/IL-17 axis to mediate the crosstalk between dendritic cells (DCs) and IL-17-producing cells [26]. Besides IL-36, IL-6, together with transforming growth factor beta (TGF $\beta$ ), have been shown to induce naive $\mathrm{CD} 4^{+} \mathrm{T}$ cells differentiation into $\mathrm{T}$ helper 17 (Th17) cells, while TNF activates DCs to produce IL-23 or induces keratinocytes to constantly produce chemokines such as chemokine (C-C-motif) ligand 20 (CCL20) and chemokine (C-X-C-motif) ligand 1 (CXCL1) to recruit immune cells to the 
site of injury [27-31], thus triggering skin inflammation in psoriasis (Figure 1). Therefore, the pathogenesis of psoriasis has once been conceptualized into an initiation by trauma (Koebner response) [17, 32].

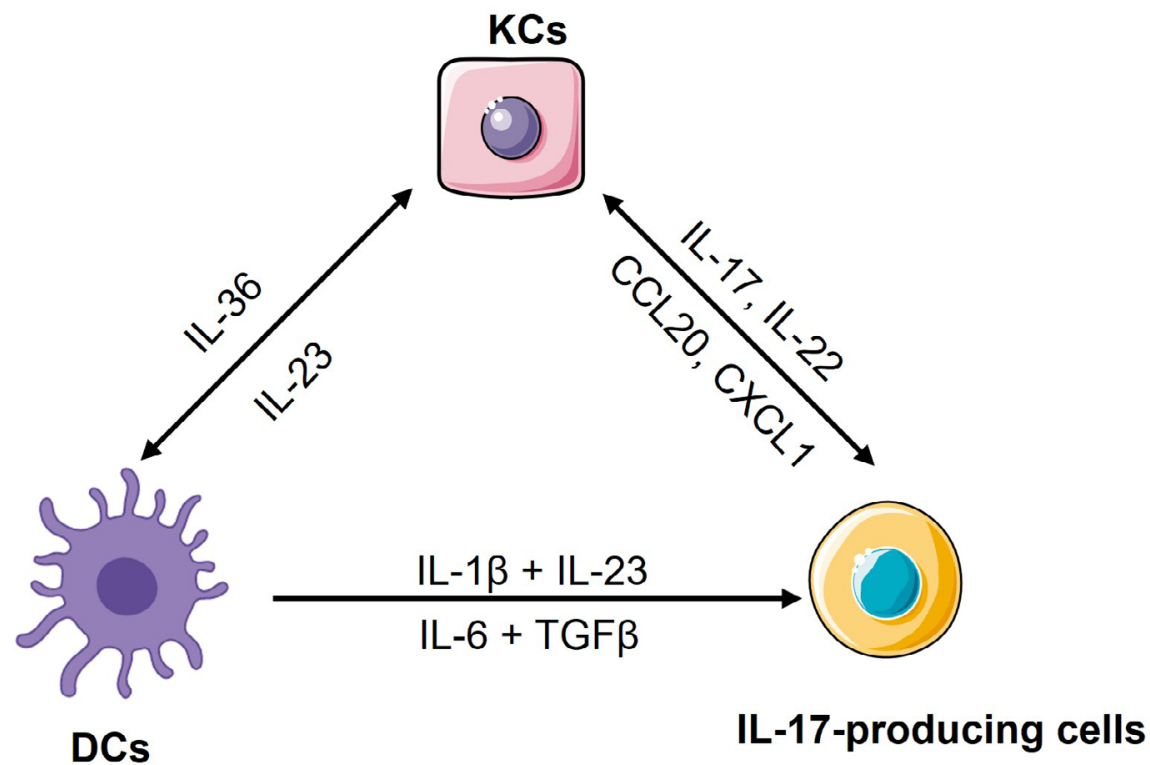

Figure 1. The crosstalk among keratinocytes, DCs and IL-17-producing cells. Upon skin injury, keratinocytes produce IL-36 to activate $\mathrm{mDC}$ s for releasing IL-1 $\beta$, IL-6 and IL-23. In mice, IL-6, together with TGF $\beta$, induce naive CD4 ${ }^{+}$T cells differentiation into Th17 cells, while in human, IL-1 $\beta$ together with IL-23, induce naive CD4 ${ }^{+} \mathrm{T}$ cells differentiation into Th17 cells. These cytokines also induce and/or activate other IL-17-producing cells to produce IL-17, IL-22 and other Th17 cytokines. Among these Th17 cytokines, IL-17 and IL-22 induce keratinocytes to produce chemokines such as CCL20 and CXCL1 for recruiting neutrophils and more IL-17-producing T cells into sites of inflammation in the skin, or induce keratinocytes to produce more IL-36, thus forming a feed-forward inflammatory loop. KCs: keratinocytes

Taken together, the above observations demonstrate that keratinocytes are capable of initiating local inflammation by recruiting and activating immune cells, and the crosstalk between keratinocytes and immune cells is critical for the initiation and progression of psoriasis.

\section{Keratinocytes are a responder of immune cells in psoriasis}

Although our group and other groups have shown that the overactivation of keratinocytes in response to skin injury can trigger psoriasis, several mechanisms indicated that hyperproliferation and aberrant differentiation of keratinocytes are secondary phenomenon induced by immune activation [33], especially in a "feed-forward" mechanism of psoriasis [34]. In this mechanism, keratinocytes have been thought as a responder of inflammatory mediators released by DCs and/or IL-17-producing cells [35]. Mechanically, in lesional skin, activated mDCs by IL-36 produce high amount of IL-1 $\beta$, IL-6 and IL-23 via unclear mechanisms [36-38]. IL-1 $\beta$ and IL-23 drive human naive CD4+ $\mathrm{T}$ cells differentiation into Th17 cell [39], while IL-6, together with TGF $\beta$, promotes murine naive CD4+ ${ }^{+}$cells development into Th17 cell [40], and then IL-23 activates Th17 cells to produce IL-17A, IL-17F, IL-22 and other cytokines [17, 18, 41-43]. Among these cytokines, IL-17 cytokines induce keratinocytes or other innate immune cells to produce a plethora of cytokines [44-46], chemokines [47] and AMPs [25, 48, 49] to promote the expansion of Th17 cells, $\gamma \delta \mathrm{T}$ cells and group 3 innate lymphoid cells (ILC3) or recruit neutrophils and more IL-17-producing T cells into sites of inflammation in the skin [26, 50-53] (Figure 1). In addition to inflammation, IL-17, IL-22 or IL-23 can maintain the acanthosis that is a hallmark of psoriasis. For example, IL-17A directly acts on keratinocytes to induce the expression of REG3A to promote keratinocyte hyperproliferation and epidermal hypoplasia [25]. Thus, the crosstalk between keratinocytes and the IL-17-producing T cells facilitates both immune and non-immune functions that drive cutaneous inflammation and epidermal hyperproliferation for developing the full-blown psoriasiform phenotype, and unceasing crosstalk between keratinocytes and immune cells comprises a positive feed-forward mechanism and further amplifies local inflammatory responses [5, 53]. The recent finding that mice with a specific deletion of IL-17 receptor A (IL-17RA) in keratinocytes, but not in $\mathrm{T}$ cells, neutrophils, or macrophages, were largely protected from the inflammatory response $[54,55]$ 
further confirms that this feed-forward inflammatory loop is critically sustained by the crosstalk between keratinocytes and immune cells via IL-17RA. All these data also suggest that targeting IL-17A/IL-17RA or IL-23 can cease the crosstalk between keratinocytes and immune cells, and would be effective treatment strategy for psoriasis patients [53]. Mounting clinical trials support the hypothesis, and show that antibodies targeting IL-17A/IL-17RA or IL-23, such as secukinumab, ixekizumab, brodalumab, tildrakizumab, guselkumab and risankizumab, have achieved tremendous success in the treatment of psoriasis $[34,53,56]$.

Although psoriasis can be successfully treated by using the above biologics to cease the crosstalk between keratinocytes and immune cells, skin lesions often recur after withdrawal of these biologics [57-60]. Memory $\mathrm{T}$ cells residing in the skin have been considered as a major driver of psoriasis relapse [61, 62]. However, how skin resident memory T cells (TRM) are activated to reinitiate inflammation in psoriasis remains largely unknown. In addition to TRM, keratinocytes with stemness from the basal layer have recently been found to gain inflammatory memory during inflammation $[63,64]$. Would keratinocytes with inflammatory memory be involved in psoriasis relapse? Can "memory" keratinocytes crosstalk with TRM to trigger psoriasis relapse? More efforts are required to address these questions.

\section{Crosstalk between keratinocytes and immune cells in AD}

$\mathrm{AD}$ is another chronic, relapsing and Th2 cell-driven inflammatory skin disorder characterized by barrier disruption, intense pruritus and eczematous lesions [65]. Therefore, Th2-type inflammation and barrier dysfunction are thought to be essential for the pathogenesis of AD [4]. So far, there are two views of $\mathrm{AD}$ pathogenesis, i.e. "inside-out" versus "outside-in" views [66]. In both views, keratinocytes are the key cell type that link barrier defects and the Th2 response in AD. Here we will summarize these two views in details.

\section{Inflammatory mediators from immune cells enforce keratinocyte defects}

Increasing evidence shows that keratinocytes from patients with AD produce increased amounts of multiple chemokines and cytokines compared with healthy cells, which mediate the crosstalk between keratinocytes and multiple immune cells. For example, compared to keratinocytes in normal patients, keratinocytes from lesional skin of $\mathrm{AD}$ patients produce the much higher level of thymic stromal lymphopoietin (TSLP) to potently activate CD11c ${ }^{+}$DCs [67]. TSLP-activated DCs, on one hand, produce the Th2-attracting chemokines, thymus and activation-regulated chemokine (TARC, also known as CCL17) and macrophage-derived chemokine (MDC, also known as CCL22) to recruit Th2 cells into lesional skin of AD; on the other hand, prime Th2 cells to produce Th2 cytokines such as IL-4, IL-5 and IL-13 [68, 69]. These Th2 cytokines either induce and amplify the inflammation in eosinophils [70] or directly induce an immunoglobulin isotype switch to immunoglobulin E (IgE) in B cells [71], and then IgE triggers mast cells and basophils to release a diverse group of biologically active products including histamine and leukotriene C4 (LTC4) [72]. Moreover, these Th2 cytokines can directly drive keratinocytes to further produce more TSLP [73] or inhibit the expression of filaggrin (FLG), a protein involved in keratinocyte differentiation [74-76], to impair barrier formation of the skin [77, 78]. In addition to TSLP, keratinocytes from lesional skin of AD highly express IL-33 and IL-25 [79, 80]. IL-33 can activate Th2 cells and ILC2s to produce type 2 cytokines through a receptor complex consisting of IL-33-specific receptor ST2 and a co-receptor IL-1 receptor accessory protein (IL-1RAcP), or actives basophils to release IL-4 and then activates ILC2 [81]. IL-25 produced by keratinocytes also has a dual role in both inducing the Th2 response and inhibiting FLG synthesis in keratinocytes [82]. Moreover, both IL-33 and IL-4 can induce Th2 cells or mast cells to release IL-31 [83, 84], and then IL-31 promotes pruritus and scratching behavior in AD [85]. Scratching the skin, in turns, further induces the expression and/or release of IL-33 and IL-25 from keratinocytes [81, 86, 87]. Therefore, the crosstalk between keratinocytes and immune cells forms a positive feedback loop to drive the pathogenesis of AD (Figure 2). This is historically referred to as "the inside-out" model of AD [88]. 


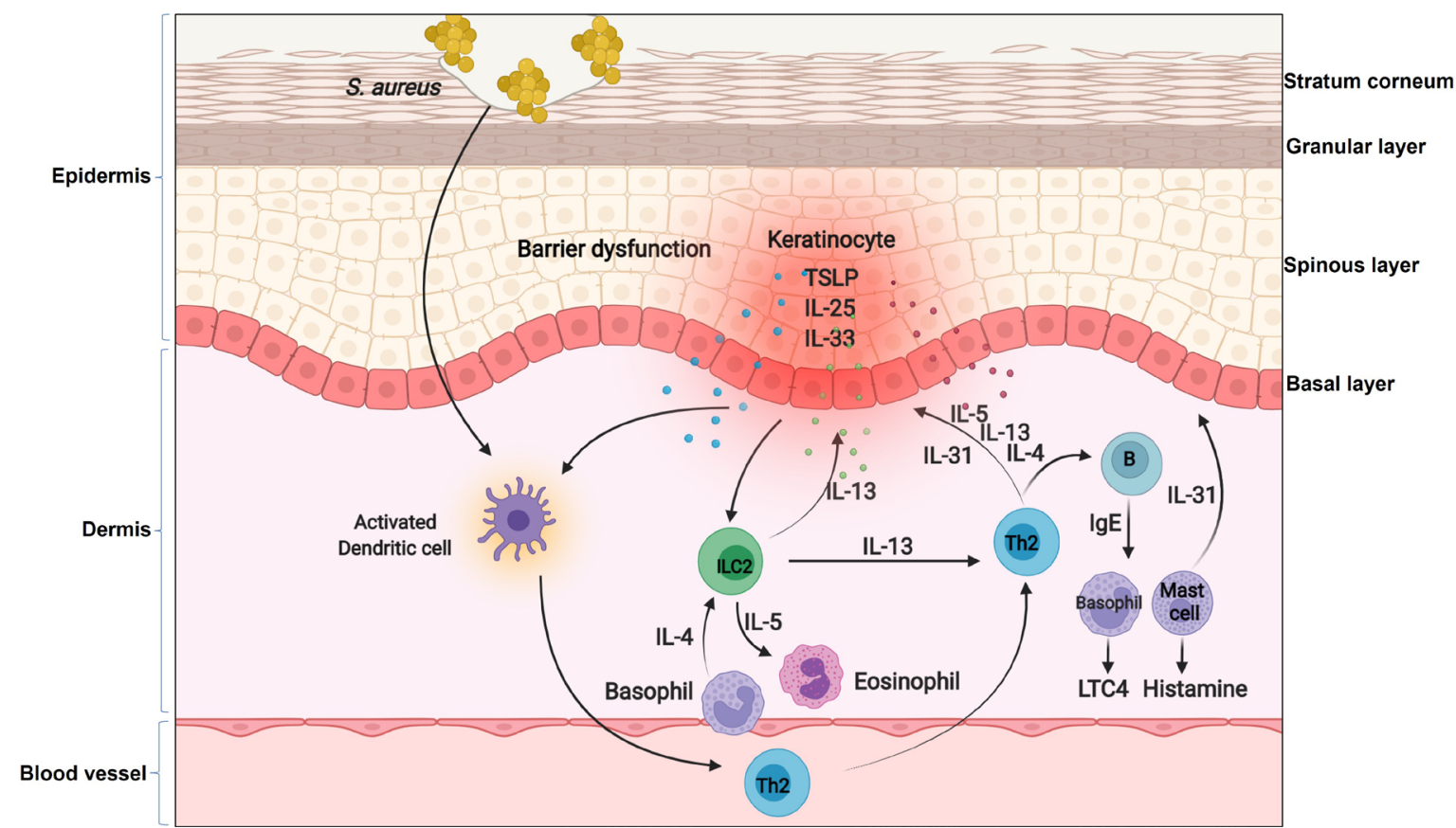

Figure 2. The inflammatory loop in AD. In the context of AD, keratinocytes produce cytokines such as TSLP, IL-25 and IL-33. These cytokines on one hand activate DCs to produce chemokines for recruiting Th2 cells and ILC2 into lesional skin, on the other hand directly active basophils to release IL-4 and then activate ILC2. Th2 cells and ILC2 produce IL-4, IL-5 and IL-13 to induce an immunoglobulin isotype switch to $\lg \mathrm{E}$ in B cells, and then $\lg \mathrm{E}$ triggers mast cells and basophils to release a diverse group of biologically active products including histamine and LTC4. Moreover, these Th2 cytokines can inhibit FLG expression in keratinocytes or further induce keratinocytes to produce TSLP. Furthermore, the genetic defects in keratinocytes impair skin barrier and facilitates Staphylococcus aureus (S. aureus) entry through the epidermal barrier. Penetrated S. aureus enables them to come direct contact with viable immune cells and enhances the expression of Th2 cytokines such as IL-4

\section{Genetic defects of keratinocyte drive dysregulated responses of immune cells}

Besides dysregulated immune responses, a defective barrier function is also a well-recognized feature of AD. Individuals with inherited aberrant production either in serine protease (SP)/ antiprotease expression or FLG usually develop moderate to severe AD [66]. The strongest evidence for a primary structural defect of $\mathrm{SC}$ involved in the pathogenesis of $\mathrm{AD}$ derives from the link between loss-of-function mutations in the gene encoding keratinocyte FLG and AD [66, 74, 89]. Mutations in FLG are common in Northern European patients with $\mathrm{AD}[66,90]$. These patients have permeability barrier abnormality, as loss of intracellular protein FLG alters keratinocyte differentiation to disrupt the organization of the extracellular lamellar bilayers and decreases SC hydration [66]. This disrupted skin barrier leads to increased penetration of cutaneous antigens [91] or dysbiosis [92] in AD, which is involved in the subsequent inflammatory loop of the skin, regardless of its being a cause or a consequence in AD. For example, a loss-of-function mutation in FLG facilitates $S$. aureus entry through the epidermal barrier. Penetrated $S$. aureus enables them to come direct contact with viable immune cells and enhances the expression of Th2 cytokines such as IL-4 [93] (Figure 2). IL-4 inhibits the expression of FLG as well as multiple AMPs, such as human beta defensin 2 (HBD2), HBD3 and LL-37 [94], thus further impairing skin barrier and defense. Moreover, S. aureus releases phenol-soluble modulin alpha (PSM $\alpha$ ) to induce the expression of IL-1 $\alpha$ and IL-36 $\alpha$ in keratinocytes [95], and this in turn stimulates $\gamma \delta$ T cells and ILC3 to produce IL-17, and then promoting skin damage and inflammation resembling AD [96, 97]. Therefore, the converging pathogenic features caused by FLG mutations lead to the development of specific strategies to restore barrier function in patients with $\mathrm{AD}$, and topical application of moisturizers or barrier repair products has been shown to correct both the permeability barrier abnormality and normalize immune dysfunction induced by the $S$. aureus penetration $[66,93]$. Altogether, these data suggest that primary inherited barrier abnormalities caused by the aberrant differentiation of keratinocytes not only stimulate downstream paracrine mechanisms that could further compromise permeability barrier function, but also cause dysregulated immune responses to drive the inflammatory loop in AD. 
From the above statements, both views of AD pathogenesis highlight the crosstalk between keratinocytes and immune cells (including basophils, mast cells, ILC2s, DCs and Th2 cells) plays a definitive role in the induction and propagation of AD inflammation. Multiple cytokines, such as IL-4 and IL-13, link this crosstalk. Therefore, targeting these cytokines and their signaling pathway would effectively treat AD. Dupilumab, an IL-4R $\alpha$ antagonist that inhibits both IL-4 and IL-13 signaling, and tralokinumab, an IL-13 inhibitor, have been approved for the treatment of AD [98, 99]. However, AD is a more complexed inflammatory skin disease with a wide spectrum of clinical phenotypes. Therefore, treatment of AD is still under great challenge. Moreover, relapse is common in AD patients, and the underlying mechanism of AD relapse needs further exploration. Whether the crosstalk between "memory" keratinocytes and resident TRM would be involved in AD relapse warrants further investigation.

\section{Crosstalk of keratinocytes and immune cells in other inflammatory skin disorders}

Besides psoriasis and AD, there are several other forms of inflammatory skin diseases, such as cutaneous lupus erythematosus (CLE), acne vulgaris, pemphigus and pyoderma gangrenosum (PG). In these skin disorders, the crosstalk between keratinocytes and immune cells also plays critical roles in the pathogenesis of diseases. Here we summarize how the crosstalk between keratinocytes and immune cells are involved in other inflammatory skin disorders.

\section{CLE}

It is known that type I IFNs-mediated inflammation plays a critical role in the development of CLE. Ultraviolet is a common trigger for CLE and induces keratinocytes to produce type I IFNא or induces keratinocyte apoptosis and necrosis [100]. The necrotic keratinocytes release DNAs, and DNAs complex with LL37 to stimulate the production of IFN $\alpha / \beta$ by pDCs. These type I IFNs further upregulate CLE-typical autoimmune nuclear autoantigens such as Sjogren's-symdrome-related antigen A (SSA, also called Ro)/Ro52 [101], and then autoantigen-specific $\mathrm{T}$ cells recognize these autoantigens to promote autoreactive B-cell activation [102].

\section{Acne vulgaris}

The commensal bacterium Cutibacterium acnes (C. acnes, formerly known as Propionibacterium acnes) has been thought as one of triggers of acne vulgaris. This bacterium can activate TLRs 2 and 4 in keratinocytes, and keratinocytes release pro-inflammatory cytokines such as IL-6 and TNF to induce innate immune and inflammatory events [103-105]. The innate immune activation of keratinocytes and the inflammatory milieu they generate in acne environment favor the activation of other cell types, including sebocytes, DCs, macrophages [106] and Th1/Th17 cells in lesions [107]. These events thus lead to the appearance of macroscopic inflammatory acne lesions in puberty.

\section{Pemphigus}

Pemphigus vulgaris (PV) is an autoimmune blistering skin disease characterized by the loss of epidermal cell-cell adhesion caused by autoantibodies targeting keratinocyte surface antigens such as desmoglein (Dsg). In this disease setting, genetic factors facilitate auto-reactive T cells to induce autoantibody production against desmosomal cadherins, thus leading to keratinocyte dissociation and blister formation [108, 109]. Moreover, PV-derived autoantibodies stimulate keratinocytes to secrete cytokines such as TNF, IL-6 and IL-1 $\alpha[110,111]$, which contribute to the activation of antigen-specific Th cells such as Th2 cells [111]. The activated antigen-specific $\mathrm{T}$ cells interact with the $\mathrm{B}$ cell to induce the production of autoantibodies, thus triggering the development of PV phenotype.

\section{PG}

PG is generally considered as an autoinflammatory skin disease with obvious neutrophil accumulation in skin lesions, although the cause of PG is not well understood. Keratinocytes play key roles in neutrophil accumulation. Because keratinocytes in PG produce high levels of the pro-inflammatory cytokines such 
as IL-1 $\alpha$, IL-1 $\beta$, IL-8, IL-36, or chemokines including CXCL9, CXCL10 and CXCL11. IL-8, a major neutrophil chemotactic factor, promotes neutrophil recruitment, while other chemokines attract Th1 and Th17 cells infiltration into the wound periphery or around adnexal structures. These T cells produce IL-17A or other cytokines that in turn act on keratinocytes to induce the expression of various chemokines to recruit neutrophil accumulation or act in concert with TNF and IL-1 $\beta$ to further drive inflammatory pathways [112].

\section{Conclusions}

In past decades, there have been enormous progresses in the understanding of the crosstalk between keratinocytes and immune cells in the pathogenesis of inflammatory skin disorders. Keratinocytes express and secrete a variety of cytokines, chemokines, and AMPs to recruit and activate almost any type of immune cells, including DCs, Th1, Th2, and Th17 cells. Activated immune cells express inflammatory mediators to enforce immune responses of keratinocytes. Thus, the crosstalk between keratinocytes and immune cells forms a "feed-forward" or "feedback" loop to sustain the chronicity of inflammatory skin diseases. The recognition of the importance of the crosstalk between keratinocytes and immune cells leads to multiple successful drugs in the treatment of inflammatory skin diseases, such as neutralizing antibodies targeting the IL-23/IL-17 axis for psoriasis treatment [113] and the neutralizing antibody inhibiting IL-4R in the treatment of $\mathrm{AD}[99,114]$. However, the relapse of skin inflammatory diseases is still out of control. Future efforts are needed to explore the underlying intricate mechanism of the relapse of skin inflammatory diseases. Since both "memory" keratinocytes and TRM contribute to psoriasis relapse and the crosstalk between keratinocytes and immune cells plays key roles in multiple inflammatory skin diseases, whether the crosstalk between "memory" keratinocytes and TRM would be a general mechanism of the relapse of inflammatory skin diseases definitely warrant further investigation.

\section{Abbreviations}

AD: atopic dermatitis

AMPs: antimicrobial peptides/proteins

CCL20: chemokine (C-C-motif) ligand 20

CLE: cutaneous lupus erythematosus

CXCL1: chemokine (C-X-C-motif) ligand 1

DCs: dendritic cells

FLG: filaggrin

IFN $\alpha$ : interferon alpha

IgE: immunoglobulin $\mathrm{E}$

IL-24: interleukin 24

IL-17RA: interleukin 17 receptor A

ILC3: group 3 innate lymphoid cells

LTC4: leukotriene C4

mDCs: myeloid dendritic cells

pDCs: plasmacytoid dendritic cells

PG: pyoderma gangrenosum

PV: pemphigus vulgaris

REG3A: regenerating islet-derived protein 3 alpha

S. aureus: Staphylococcus aureus

SC: stratum corneum

TGF $\beta$ : transforming growth factor beta 
Th17: T helper 17

TLR9: Toll-like receptor 9

TNF: tumor necrosis factor

TRM: resident memory T cells

TSLP: thymic stromal lymphopoietin

\section{Declarations}

\section{Author contributions}

YL conceived the manuscript. YL and XN wrote the manuscript, and YL and XN edited the manuscript.

\section{Conflicts of interest}

The authors declare that they have no conflicts of interest.

\section{Ethical approval}

Not applicable.

\section{Consent to participate}

Not applicable.

\section{Consent to publication}

Not applicable.

Availability of data and materials

Not applicable.

\section{Funding}

The work in Yuping Lai's group is supported by National Natural Science Foundation of China (82071785 \& 31670925), National Key Research and Development Program of China (2016YFC0906200 \& 2016YFC0906202 to YL), and ECNU Multifunctional Platform for Innovation (011). The funders had no role in study design, data collection and analysis, decision to publish, or preparation of the manuscript.

\section{Copyright}

(c) The Author(s) 2021.

\section{References}

1. Jiang Y, Tsoi LC, Billi AC, Ward NL, Harms PW, Zeng C, et al. Cytokinocytes: the diverse contribution of keratinocytes to immune responses in skin. JCI Insight. 2020;5:e142067.

2. Eckert RL, Efimova T, Dashti SR, Balasubramanian S, Deucher A, Crish JF, et al. Keratinocyte survival, differentiation, and death: many roads lead to mitogen-activated protein kinase. J Investig Dermatol Symp Proc. 2002;7:36-40.

3. Raj D, Brash DE, Grossman D. Keratinocyte apoptosis in epidermal development and disease. J Invest Dermatol. 2006;126:243-57.

4. Dainichi T, Kitoh A, Otsuka A, Nakajima S, Nomura T, Kaplan DH, et al. The epithelial immune microenvironment (EIME) in atopic dermatitis and psoriasis. Nat Immunol. 2018;19:1286-98.

5. Ni X, Lai Y. Keratinocyte: a trigger or an executor of psoriasis? J Leukoc Biol. 2020;108:485-91.

6. $\quad$ Nickoloff BJ. The cytokine network in psoriasis. Arch Dermatol. 1991;127:871-84.

7. Barker JN, Mitra RS, Griffiths CE, Dixit VM, Nickoloff BJ. Keratinocytes as initiators of inflammation. Lancet. 1991;337:211-4. 
8. Uyemura K, Yamamura M, Fivenson DF, Modlin RL, Nickoloff BJ. The cytokine network in lesional and lesion-free psoriatic skin is characterized by a T-helper type 1 cell-mediated response. J Invest Dermatol. 1993;101:701-5.

9. Pasparakis M, Courtois G, Hafner M, Schmidt-Supprian M, Nenci A, Toksoy A, et al. TNF-mediated inflammatory skin disease in mice with epidermis-specific deletion of IKK2. Nature. 2002;417:861-6.

10. Kumari S, Bonnet MC, Ulvmar MH, Wolk K, Karagianni N, Witte E, et al. Tumor necrosis factor receptor signaling in keratinocytes triggers interleukin-24-dependent psoriasis-like skin inflammation in mice. Immunity. 2013;39:899-911.

11. Zenz R, Eferl R, Kenner L, Florin L, Hummerich L, Mehic D, et al. Psoriasis-like skin disease and arthritis caused by inducible epidermal deletion of Jun proteins. Nature. 2005;437:369-75.

12. Garber K. Genetics: deep exploration. Nature. 2012;492:S56-7.

13. Jordan CT, Cao L, Roberson ED, Pierson KC, Yang CF, Joyce CE, et al. PSORS2 is due to mutations in CARD14. Am J Hum Genet. 2012;90:784-95.

14. Perera GK, Di Meglio P, Nestle FO. Psoriasis. Annu Rev Pathol. 2012;7:385-422.

15. Lee YJ, Kim SJ, Moon TS. Multilevel regulation of bacterial gene expression with the combined STAR and antisense RNA system. ACS Synth Biol. 2018;7:853-65.

16. Wang M, Zhang S, Zheng G, Huang J, Songyang Z, Zhao X, et al. Gain-of-function mutation of Card14 leads to spontaneous psoriasis-like skin inflammation through enhanced keratinocyte response to IL-17A. Immunity. 2018;49:66-79.e5.

17. Lande R, Gregorio J, Facchinetti V, Chatterjee B, Wang YH, Homey B, et al. Plasmacytoid dendritic cells sense self-DNA coupled with antimicrobial peptide. Nature. 2007;449:564-9.

18. Ganguly D, Chamilos G, Lande R, Gregorio J, Meller S, Facchinetti V, et al. Self-RNA-antimicrobial peptide complexes activate human dendritic cells through TLR7 and TLR8. J Exp Med. 2009;206:1983-94.

19. Li N, Yamasaki K, Saito R, Fukushi-Takahashi S, Shimada-Omori R, Asano M, et al. Alarmin function of

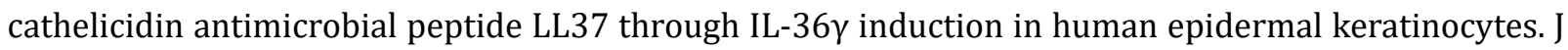
Immunol. 2014;193:5140-8.

20. Girolomoni G, Strohal R, Puig L, Bachelez H, Barker J, Boehncke WH, et al. The role of IL-23 and the IL-23/ $\mathrm{T}_{\mathrm{H}} 17$ immune axis in the pathogenesis and treatment of psoriasis. J Eur Acad Dermatol Venereol. 2017;31:1616-26.

21. Lowes MA, Russell CB, Martin DA, Towne JE, Krueger JG. The IL-23/T17 pathogenic axis in psoriasis is amplified by keratinocyte responses. Trends Immunol. 2013;34:174-81.

22. Albanesi C, Madonna S, Gisondi P, Girolomoni G. The interplay between keratinocytes and immune cells in the pathogenesis of psoriasis. Front Immunol. 2018;9:1549.

23. Lai Y, Di Nardo A, Nakatsuji T, Leichtle A, Yang Y, Cogen AL, et al. Commensal bacteria regulate Toll-like receptor 3-dependent inflammation after skin injury. Nat med. 2009;15:1377-82.

24. Jiang Z, Liu Y, Li C, Chang L, Wang W, Wang Z, et al. IL-36 $\gamma$ induced by the TLR3-SLUG-VDR axis promotes wound healing via REG3A. J Invest Dermatol. 2017;137:2620-9.

25. Lai Y, Li D, Li C, Muehleisen B, Radek KA, Park HJ, et al. The antimicrobial protein REG3A regulates keratinocyte proliferation and differentiation after skin injury. Immunity. 2012;37:74-84.

26. Tortola L, Rosenwald E, Abel B, Blumberg H, Schäfer M, Coyle AJ, et al. Psoriasiform dermatitis is driven by IL-36-mediated DC-keratinocyte crosstalk. J Clin Invest. 2012;122:3965-76.

27. Kennedy-Crispin M, Billick E, Mitsui H, Gulati N, Fujita H, Gilleaudeau P, et al. Human keratinocytes' response to injury upregulates CCL20 and other genes linking innate and adaptive immunity. J Invest Dermatol. 2012;132:105-13.

28. Morales J, Homey B, Vicari AP, Hudak S, Oldham E, Hedrick J, et al. CTACK, a skin-associated chemokine that preferentially attracts skin-homing memory T cells. Proc Natl Acad Sci U S A. 1999;96:14470-5. 
29. Homey B, Alenius H, Muller A, Soto H, Bowman EP, Yuan W, et al. CCL27-CCR10 interactions regulate T cell-mediated skin inflammation. Nat Med. 2002;8:157-65.

30. Russo C, Polosa R. TNF-alpha as a promising therapeutic target in chronic asthma: a lesson from rheumatoid arthritis. Clin Sci (Lond). 2005;109:135-42.

31. Aggarwal BB, Shishodia S, Takada Y, Jackson-Bernitsas D, Ahn KS, Sethi G, et al. TNF blockade: an inflammatory issue. Ernst Schering Res Found Workshop. 2006;56:161-86.

32. Gilliet M, Lande R. Antimicrobial peptides and self-DNA in autoimmune skin inflammation. Curr Opin Immunol. 2008;20:401-7.

33. Lowes MA, Suarez-Farinas M, Krueger JG. Immunology of psoriasis. Annu Rev Immunol. 2014;32:227-55.

34. Hawkes JE, Chan TC, Krueger JG. Psoriasis pathogenesis and the development of novel targeted immune therapies. J Allergy Clin Immunol. 2017;140:645-53.

35. Harper EG, Guo C, Rizzo H, Lillis JV, Kurtz SE, Skorcheva I, et al. Th17 cytokines stimulate CCL20 expression in keratinocytes in vitro and in vivo: implications for psoriasis pathogenesis. J Invest Dermatol. 2009;129:2175-83.

36. Furue K, Yamamura K, Tsuji G, Mitoma C, Uchi H, Nakahara T, et al. Highlighting interleukin-36 signalling in plaque psoriasis and pustular psoriasis. Acta Derm Venereol. 2018;98:5-13.

37. Gabay C, Towne JE. Regulation and function of interleukin-36 cytokines in homeostasis and pathological conditions. J Leukoc Biol. 2015;97:645-52.

38. Foster AM, Baliwag J, Chen CS, Guzman AM, Stoll SW, Gudjonsson JE, et al. IL-36 promotes myeloid cell infiltration, activation, and inflammatory activity in skin. J Immunol. 2014;192:6053-61.

39. Revu S, Wu J, Henkel M, Rittenhouse N, Menk A, Delgoffe GM, et al. IL-23 and IL-1 $\beta$ drive human Th17 cell differentiation and metabolic reprogramming in absence of CD28 costimulation. Cell Rep. 2018;22:2642-53.

40. Qin H, Wang L, Feng T, Elson CO, Niyongere SA, Lee SJ, et al. TGF-beta promotes Th17 cell development through inhibition of SOCS3. J Immunol. 2009;183:97-105.

41. Hue S, Ahern P, Buonocore S, Kullberg MC, Cua DJ, McKenzie BS, et al. Interleukin-23 drives innate and T cell-mediated intestinal inflammation. J Exp Med. 2006;203:2473-83.

42. Blauvelt A, Chiricozzi A. The immunologic role of IL-17 in psoriasis and psoriatic arthritis pathogenesis. Clin Rev Allergy Immunol. 2018;55:379-90.

43. Villanova F, Flutter B, Tosi I, Grys K, Sreeneebus H, Perera GK, et al. Characterization of innate lymphoid cells in human skin and blood demonstrates increase of NKp44+ ILC3 in psoriasis. J Invest Dermatol. 2014;134:984-91.

44. Xu X, Li P, Zhang P, Chu M, Liu H, Chen X, et al. Differential effects of Rhodiola rosea on regulatory T cell differentiation and interferon- $\gamma$ production in vitro and in vivo. Mol Med Rep. 2016;14:529-36.

45. Su Y, Huang J, Zhao X, Lu H, Wang W, Yang XO, et al. Interleukin-17 receptor D constitutes an alternative receptor for interleukin-17A important in psoriasis-like skin inflammation. Sci Immunol. 2019;4:eaau9657.

46. Carrier Y, Ma HL, Ramon HE, Napierata L, Small C, O’Toole M, et al. Inter-regulation of Th17 cytokines and the IL-36 cytokines in vitro and in vivo: implications in psoriasis pathogenesis. J Invest Dermatol. 2011;131:2428-37.

47. Chiricozzi A, Guttman-Yassky E, Suarez-Farinas M, Nograles KE, Tian S, Cardinale I, et al. Integrative responses to IL-17 and TNF- $\alpha$ in human keratinocytes account for key inflammatory pathogenic circuits in psoriasis. J Invest Dermatol. 2011;131:677-87. 
48. Hegyi Z, Zwicker S, Bureik D, Peric M, Koglin S, Batycka-Baran A, et al. Vitamin D analog calcipotriol suppresses the Th17 cytokine-induced proinflammatory S100 "alarmins" psoriasin (S100A7) and koebnerisin (S100A15) in psoriasis. J Invest Dermatol. 2012;132:1416-24.

49. Liang SC, Tan XY, Luxenberg DP, Karim R, Dunussi-Joannopoulos K, Collins M, et al. Interleukin (IL)-22 and IL-17 are coexpressed by Th17 cells and cooperatively enhance expression of antimicrobial peptides. J Exp Med. 2006;203:2271-9.

50. Cai Y, Shen X, Ding C, Qi C, Li K, Li X, et al. Pivotal role of dermal IL-17-producing $\gamma \delta$ T cells in skin inflammation. Immunity. 2011;35:596-610.

51. Ha HL, Wang H, Pisitkun P, Kim JC, Tassi I, Tang W, et al. IL-17 drives psoriatic inflammation via distinct, target cell-specific mechanisms. Proc Natl Acad Sci U S A. 2014;111:E3422-31.

52. Song J, Wang H, Ng TI, Cui A, Zhu S, Huang Y, et al. Sequence analysis of the fusion protein gene of human respiratory syncytial virus circulating in China from 2003 to 2014. Sci Rep. 2018;8:17618.

53. Lai Y, Dong C. Therapeutic antibodies that target inflammatory cytokines in autoimmune diseases. Int Immunol. 2016;28:181-8.

54. Moos S, Mohebiany AN, Waisman A, Kurschus FC. Imiquimod-induced psoriasis in mice depends on the IL-17 signaling of keratinocytes. J Invest Dermatol. 2019;139:1110-7.

55. Garzorz-Stark N, Eyerich K. Psoriasis pathogenesis: keratinocytes are back in the spotlight. J Invest Dermatol. 2019;139:995-6.

56. Jullien D, Prinz JC, Nestle FO. Immunogenicity of biotherapy used in psoriasis: the science behind the scenes. J Invest Dermatol. 2015;135:31-8.

57. Masson Regnault M, Konstantinou MP, Khemis A, Poulin Y, Bourcier M, Amelot F, et al. Early relapse of psoriasis after stopping brodalumab: a retrospective cohort study in 77 patients. J Eur Acad Dermatol Venereol. 2017;31:1491-6.

58. Blauvelt A, Leonardi CL, Gooderham M, Papp KA, Philipp S, Wu JJ, et al. Efficacy and safety of continuous risankizumab therapy $v s$ treatment withdrawal in patients with moderate to severe plaque psoriasis: a phase 3 randomized clinical trial. JAMA Dermatol. 2020;156:649-58.

59. Warren RB, Carrascosa JM, Fumero E, Schoenenberger A, Lebwohl MG, Szepietowski JC, et al. Time to relapse after tildrakizumab withdrawal in patients with moderate-to-severe psoriasis who were responders at week 28: post hoc analysis through 64 weeks from reSURFACE 1 trial. J Eur Acad Dermatol Venereol. 2021;35:919-27.

60. Kimball AB, Papp KA, Reich K, Gooderham M, Li Q, Cichanowitz N, et al. Efficacy and safety of tildrakizumab for plaque psoriasis with continuous dosing, treatment interruption, dose adjustments and switching from etanercept: results from phase III studies. Br J Dermatol. 2020;182:1359-68.

61. Owczarczyk Saczonek A, Krajewska-Wlodarczyk M, Kasprowicz-Furmanczyk M, Placek W. Immunological memory of psoriatic lesions. Int J Mol Sci. 2020;21:625.

62. Matos TR, O'Malley JT, Lowry EL, Hamm D, Kirsch IR, Robins HS, et al. Clinically resolved psoriatic lesions contain psoriasis-specific IL-17-producing $\alpha \beta$ T cell clones. J Clin Invest. 2017;127:4031-41.

63. Naik S, Larsen SB, Gomez NC, Alaverdyan K, Sendoel A, Yuan S, et al. Inflammatory memory sensitizes skin epithelial stem cells to tissue damage. Nature. 2017;550:475-80.

64. Larsen SB, Cowley CJ, Sajjath SM, Barrows D, Yang Y, Carroll TS, et al. Establishment, maintenance, and recall of inflammatory memory. Cell Stem Cell. 2021;28:1758-74.e8.

65. Bieber T. Atopic dermatitis. N Engl J Med. 2008;358:1483-94.

66. Elias PM, Hatano Y, Williams ML. Basis for the barrier abnormality in atopic dermatitis: outside-inside-outside pathogenic mechanisms. J Allergy Clin Immunol. 2008;121:1337-43.

67. Ito T, Wang YH, Duramad O, Hori T, Delespesse GJ, Watanabe N, et al. TSLP-activated dendritic cells induce an inflammatory T helper type 2 cell response through OX40 ligand. J Exp Med. 2005;202:1213-23. 
68. Soumelis V, Reche PA, Kanzler H, Yuan W, Edward G, Homey B, et al. Human epithelial cells trigger dendritic cell mediated allergic inflammation by producing TSLP. Nat Immunol. 2002;3:673-80.

69. Kitajima M, Ziegler SF. Cutting edge: identification of the thymic stromal lymphopoietin-responsive dendritic cell subset critical for initiation of type 2 contact hypersensitivity. J Immunol. 2013;191:4903-7.

70. Pelaia C, Paoletti G, Puggioni F, Racca F, Pelaia G, Canonica GW, et al. Interleukin-5 in the pathophysiology of severe asthma. Front Physiol. 2019;10:1514.

71. Punnonen J, Yssel H, de Vries JE. The relative contribution of IL-4 and IL-13 to human IgE synthesis induced by activated CD4+ ${ }^{+}$or CD8 ${ }^{+}$T cells. J Allergy Clin Immunol. 1997;100:792-801.

72. Galli SJ, Tsai M. IgE and mast cells in allergic disease. Nat Med. 2012;18:693-704.

73. Bogiatzi SI, Fernandez I, Bichet JC, Marloie-Provost MA, Volpe E, Sastre X, et al. Cutting edge: proinflammatory and Th2 cytokines synergize to induce thymic stromal lymphopoietin production by human skin keratinocytes. J Immunol. 2007;178:3373-7.

74. Palmer CN, Irvine AD, Terron-Kwiatkowski A, Zhao Y, Liao H, Lee SP, et al. Common loss-of-function variants of the epidermal barrier protein filaggrin are a major predisposing factor for atopic dermatitis. Nat Genet. 2006;38:441-6.

75. Weidinger S, Illig T, Baurecht H, Irvine AD, Rodriguez E, Diaz-Lacava A, et al. Loss-of-function variations within the filaggrin gene predispose for atopic dermatitis with allergic sensitizations. J Allergy Clin Immunol. 2006;118:214-9.

76. Marenholz I, Nickel R, Ruschendorf F, Schulz F, Esparza-Gordillo J, Kerscher T, et al. Filaggrin loss-of-function mutations predispose to phenotypes involved in the atopic march. J Allergy Clin Immunol. 2006;118:866-71.

77. Furue M. Regulation of filaggrin, loricrin, and involucrin by IL-4, IL-13, IL-17A, IL-22, AHR, and NRF2: pathogenic implications in atopic dermatitis. Int J Mol Sci. 2020;21:5382.

78. Egawa G, Kabashima K. Multifactorial skin barrier deficiency and atopic dermatitis: essential topics to prevent the atopic march. J Allergy Clin Immunol. 2016;138:350-8.e1.

79. Savinko T, Matikainen S, Saarialho-Kere U, Lehto M, Wang G, Lehtimaki S, et al. IL-33 and ST2 in atopic dermatitis: expression profiles and modulation by triggering factors. J Invest Dermatol. 2012;132:1392-400.

80. Leyva-Castillo JM, Galand C, Mashiko S, Bissonnette R, McGurk A, Ziegler SF, et al. ILC2 activation by keratinocyte-derived IL-25 drives IL-13 production at sites of allergic skin inflammation. J Allergy Clin Immunol. 2020;145:1606-14.e4.

81. Imai Y. Interleukin-33 in atopic dermatitis. J Dermatol Sci. 2019;96:2-7.

82. Hvid M, Vestergaard C, Kemp K, Christensen GB, Deleuran B, Deleuran M. IL-25 in atopic dermatitis: a possible link between inflammation and skin barrier dysfunction? J Invest Dermatol. 2011;131:150-7.

83. Maier E, Werner D, Duschl A, Bohle B, Horejs-Hoeck J. Human Th2 but not Th9 cells release IL-31 in a STAT6/NF-кB-dependent way. J Immunol. 2014;193:645-54.

84. Petra AI, Tsilioni I, Taracanova A, Katsarou-Katsari A, Theoharides TC. Interleukin 33 and interleukin 4 regulate interleukin 31 gene expression and secretion from human laboratory of allergic diseases 2 mast cells stimulated by substance P and/or immunoglobulin E. Allergy Asthma Proc. 2018;39:153-60.

85. Datsi A, Steinhoff M, Ahmad F, Alam M, Buddenkotte J. Interleukin-31: the "itchy" cytokine in inflammation and therapy. Allergy. 2021;76:2982-97.

86. Cherrier M, Cerf-Bensussan N. Scratching beneath the surface: linking skin pathology with food allergy. Immunity. 2019;50:1124-6.

87. Hammad H, Lambrecht BN. Barrier epithelial cells and the control of type 2 immunity. Immunity. 2015;43:29-40. 
88. Silverberg NB, Silverberg JI. Inside out or outside in: does atopic dermatitis disrupt barrier function or does disruption of barrier function trigger atopic dermatitis? Cutis. 2015;96:359-61.

89. Smith FJ, Irvine AD, Terron-Kwiatkowski A, Sandilands A, Campbell LE, Zhao Y, et al. Loss-of-function mutations in the gene encoding filaggrin cause ichthyosis vulgaris. Nat Genet. 2006;38:337-42.

90. Cascella R, Foti Cuzzola V, Lepre T, Galli E, Moschese V, Chini L, et al. Full sequencing of the FLG gene in Italian patients with atopic eczema: evidence of new mutations, but lack of an association. J Invest Dermatol. 2011;131:982-4.

91. Horimukai K, Morita K, Narita M, Kondo M, Kitazawa H, Nozaki M, et al. Application of moisturizer to neonates prevents development of atopic dermatitis. J Allergy Clin Immunol. 2014;134:824-30.e6.

92. Byrd AL, Deming C, Cassidy SKB, Harrison OJ, Ng WI, Conlan S, et al. Staphylococcus aureus and Staphylococcus epidermidis strain diversity underlying pediatric atopic dermatitis. Sci Transl Med. 2017;9:eaal4651.

93. Nakatsuji T, Chen TH, Two AM, Chun KA, Narala S, Geha RS, et al. Staphylococcus aureus exploits epidermal barrier defects in atopic dermatitis to trigger cytokine expression. J Invest Dermatol. 2016;136:2192-200.

94. Nomura I, Goleva E, Howell MD, Hamid QA, Ong PY, Hall CF, et al. Cytokine milieu of atopic dermatitis, as compared to psoriasis, skin prevents induction of innate immune response genes. J Immunol. 2003;171:3262-9.

95. Syed AK, Reed TJ, Clark KL, Boles BR, Kahlenberg JM. Staphlyococcus aureus phenol-soluble modulins stimulate the release of proinflammatory cytokines from keratinocytes and are required for induction of skin inflammation. Infect Immun. 2015;83:3428-37.

96. Nakagawa S, Matsumoto M, Katayama Y, Oguma R, Wakabayashi S, Nygaard T, et al. Staphylococcus aureus virulent PSM $\alpha$ peptides induce keratinocyte alarmin release to orchestrate IL-17-dependent skin inflammation. Cell Host Microbe. 2017;22:667-77.e5.

97. Liu H, Archer NK, Dillen CA, Wang Y, Ashbaugh AG, Ortines RV, et al. Staphylococcus aureus epicutaneous exposure drives skin inflammation via IL-36-mediated T cell responses. Cell Host Microbe. 2017;22:653-66.e5.

98. Gooderham MJ, Hong HC, Eshtiaghi P, Papp KA. Dupilumab: a review of its use in the treatment of atopic dermatitis. J Am Acad Dermatol. 2018;78:S28-36.

99. Bieber T. Atopic dermatitis: an expanding therapeutic pipeline for a complex disease. Nat Rev Drug Discov. 2021; [Epub ahead of print].

100. Little AJ, Vesely MD. Cutaneous lupus erythematosus: current and future pathogenesis-directed therapies. Yale J Biol Med. 2020;93:81-95.

101. Wenzel J. Cutaneous lupus erythematosus: new insights into pathogenesis and therapeutic strategies. Nat Rev Rheumatol. 2019;15:519-32.

102. Bosch X. Systemic lupus erythematosus and the neutrophil. N Engl J Med. 2011;365:758-60.

103. Xia X, Li Z, Liu K, Wu Y, Jiang D, Lai Y. Staphylococcal LTA-induced miR-143 inhibits propionibacterium acnes-mediated inflammatory response in skin. J Invest Dermatol. 2016;136:621-30.

104. Kim J, Ochoa MT, Krutzik SR, Takeuchi O, Uematsu S, Legaspi AJ, et al. Activation of toll-like receptor 2 in acne triggers inflammatory cytokine responses. J Immunol. 2002;169:1535-41.

105. Pivarcsi A, Bodai L, Rethi B, Kenderessy-Szabo A, Koreck A, Szell M, et al. Expression and function of Toll-like receptors 2 and 4 in human keratinocytes. Int Immunol. 2003;15:721-30.

106. Mattii M, Lovaszi M, Garzorz N, Atenhan A, Quaranta M, Lauffer F, et al. Sebocytes contribute to skin inflammation by promoting the differentiation of T helper 17 cells. Br J Dermatol. 2018;178:722-30.

107. Kistowska M, Meier B, Proust T, Feldmeyer L, Cozzio A, Kuendig T, et al. Propionibacterium acnes promotes Th17 and Th17/Th1 responses in acne patients. J Invest Dermatol. 2015;135:110-8. 
108. Spindler V, Eming R, Schmidt E, Amagai M, Grando S, Jonkman MF, et al. Mechanisms causing loss of keratinocyte cohesion in pemphigus. J Invest Dermatol. 2018;138:32-7.

109. Walter E, Vielmuth F, Wanuske MT, Seifert M, Pollmann R, Eming R, et al. Role of Dsg1- and Dsg3-mediated signaling in pemphigus autoantibody-induced loss of keratinocyte cohesion. Front Immunol. 2019;10:1128.

110. Vodo D, Sarig O, Geller S, Ben-Asher E, Olender T, Bochner R, et al. Identification of a functional risk variant for pemphigus vulgaris in the ST18 gene. PLoS Genet. 2016;12:e1006008.

111. Giordano CN, Sinha AA. Cytokine networks in pemphigus vulgaris: an integrated viewpoint. Autoimmunity. 2012;45:427-39.

112. Maverakis E, Marzano AV, Le ST, Callen JP, Bruggen MC, Guenova E, et al. Pyoderma gangrenosum. Nat Rev Dis Primers. 2020;6:81.

113. Hawkes JE, Yan BY, Chan TC, Krueger JG. Discovery of the IL-23/IL-17 signaling pathway and the treatment of psoriasis. J Immunol. 2018;201:1605-13.

114. Harb H, Chatila TA. Mechanisms of dupilumab. Clin Exp Allergy. 2020;50:5-14. 